\title{
Single-Step Fabrication of Multispectral Filter Arrays Using Grayscale Lithography and Metal-Insulator-Metal Geometry
}

\author{
Calum Williams $^{1 *}$, George S.D. Gordon ${ }^{1}$, Timothy D. Wilkinson ${ }^{1}$, Sarah E. Bohndiek ${ }^{2}$ \\ ${ }^{\prime}$ Electrical Engineering Division, Department of Engineering, University of Cambridge, 9 JJ Thomson Avenue, Cambridge CB3 OFA, UK \\ ${ }^{2}$ Department of Physics, Cavendish Laboratory, University of Cambridge, JJ Thomson Avenue, Cambridge, CB3 OHE, UK \\ *corresponding author: cw507@cam.ac.uk
}

\begin{abstract}
Metal-insulator-metal geometries can provide optical transmission filtering, with peak wavelength dependent on insulator thickness. Using grayscale electron beam lithography to control insulator thickness, we fabricate multispectral filter arrays, whereby dose determines wavelength.
\end{abstract}

OCIS codes: (220.4000) Microstructure fabrication, (110.4234) Multispectral and hyperspectral imaging, (310.0310) Thin films

Image sensors based on silicon typically exhibit a broadband spectral response. Color filter arrays (CFAs) are thinfilm mosaics of optical filters, which when integrated atop image sensor pixels provide image data resolved into several different wavelengths. The ubiquitous Bayer CFA [1] consists of a mosaic with separate red, green and blue (RGB) filters, replicating the color information that can be resolved by our eyes for imaging. Additional filter elements can be integrated into a mosaic to provide additional wavelength detection bands albeit at the expense of spatial resolution. Multispectral filter arrays (MSFAs) and thus multispectral imaging (MSI) have recently been integrated in research fields as diverse as astronomy and biomedical imaging [2].

To fabricate MSFAs, absorptive dyes / pigments or many-layer Bragg stacks are generally implemented [2]. Both methods can be cumbersome from fabrication perspective, requiring N-lithographic steps for an N-wavelength MSFA. The continual shrinking of pixel dimensions with increasingly complex mosaics means fabrication becomes technically challenging and expensive. Additionally, for N-wavelengths, N-material compositions - either dyes or combinations of alternating dielectrics in the Bragg stack - are required [2]. Recently, nanophotonic approaches to fabricating MSFAs have been more widely explored, particularly exploiting metallic (plasmonic), or all-dielectric, nanostructure arrays / metasurfaces [3-5]. At present, however, widespread application of MSFAs based on these technologies are limited by undesirable characteristics such as low transmission, broad spectral responses and /or multi-order resonances based on their in-plane periodicity.

Here, we experimentally demonstrate a single-step fabrication methodology for high transmission efficiency, narrow bandwidth MSFAs that are able to filter light across the visible to near-infrared spectral ranges. Grayscale electron beam lithography (EBL), combined with metal-insulator metal (MIM) geometry and low critical dose negative photoresist, is used for in-plane spatially variant wavelength filtering. MIM geometries exhibit strong Fabry-Perot resonances, whose spectral position is a function of insulator thickness [6]. Thickness variation in the insulator (photoresist) is generated through grayscale EBL, determined through optimization of exposure dose and specific processing conditions.

EBL grayscale dose determines resist thickness variation, which in turn delivers optical transmission filtering through MIM geometry (Fig.1a). The MIM MSFAs consist of a $\mathrm{SiO}_{2}$ substrate with two ultrathin Ag layers encapsulating the resist, with a $\mathrm{MgF}_{2}$ coating providing a passivation layer. $\mathrm{MaN}-2400$ series negative tone photoresist is utilized for this study due to its relatively high sensitivity and resolution capability. High voltage (80kV), high current (1.5nA) EBL (Nanobeam Ltd.) is used for the patterning, and thermal evaporation is used for thin-film material deposition. The resist's sensitivity curve (Fig.1a,i) is experimentally determined through dose tests, yielding dose-to-remaining resist thickness $\left(t_{R}\right)$ information, i.e. grayscale values. Here, a dose range of $10-70 \mu \mathrm{C} . \mathrm{cm}^{-2}$ is imparted into the resist with spun thickness $\sim 350 \mathrm{~nm}$ (Fig.1a,ii), and developed in AZ-MIF-726 developer solution. Depositing a second Ag layer, post-development, yields varying height MIM structures (Fig.1a,iii), capable of filtering light (Fig.1a,iv), according to the excitation of Fabry-Perot resonant modes in the MIM cavity. 
(a)

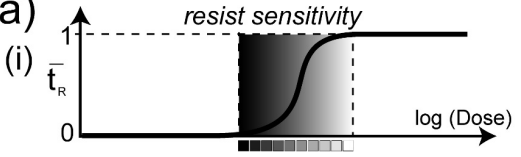

(ii)

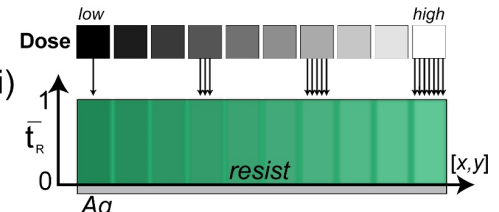

(iii)

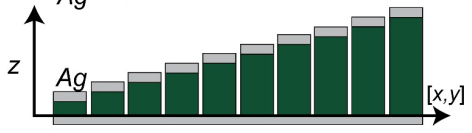

(iv)

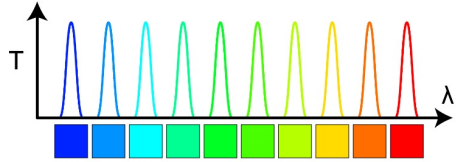

(b)

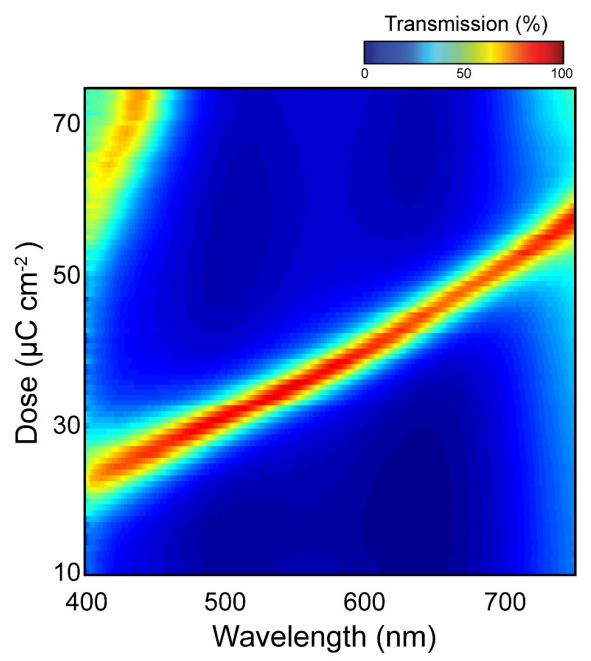

(c)

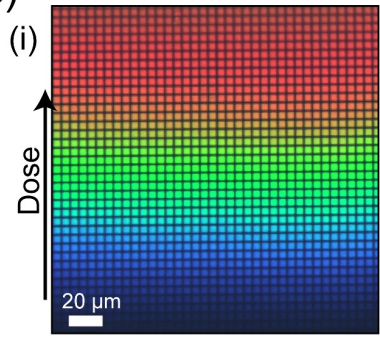

(ii)

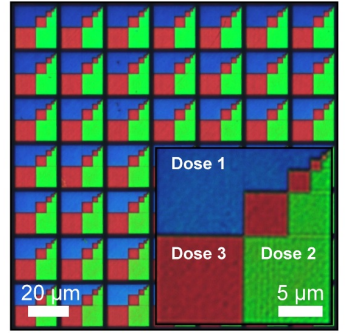

Fig. 1. Grayscale EBL to produce mosaicked CFAs: (a) Concept: (i) Typical normalized negative tone photoresist sensitivity curve. A dosemodulated (grayscale) pattern, is imparted into the photoresist atop of a Ag layer (ii), it is developed, resulting in thickness variation in $[z]$ (iii). Depositing a second Ag layer results in varying height MIM structures, which can be used for transmission (T) filtering (iv). (b) Experimental transmission spectra of an array of exposed squares with different doses $\left(10-70 \mu \mathrm{C} . \mathrm{cm}^{-2}\right)$; both first and second order resonant modes are observed. (c) Two fabricated dose-modulated samples in transmission under an optical microscope: (i) An example of the array used for characterization in (b), and (ii) RGB CFA array of decreasing dimensions.

The experimental transmission spectra (Fig.1b) for a typical dose-modulated (grayscale) MIM pattern are measured using an optical microscope (Olympus BX-51) connected via multi-mode optical fiber to a spectrometer (Ocean Optics HR2000+). The transmission maxima that can be achieved using this method is shown to range from 400 to $750 \mathrm{~nm}$ as the exposure dose increases, corresponding to a thicker insulator layer, as expected. Only the first-order resonance is present at lower doses, but for higher doses, the second-order mode is apparent resulting in dual-peak behavior. Maximum transmission of $\sim 75 \%$ and narrow FHWMs of $\sim 50 \mathrm{~nm}$ are observed. The linewidths can be further reduced by optimizing the Ag mirror thicknesses. Two examples of dose-modulated MIM patterns on glass were fabricated and imaged under the optical microscope (Fig.1c, transmission). A one-dimensionally varying dose pattern encoded with square pixels (Fig.1c,i) is the source of the spectra in Fig.1b. A simple RGB MSFA with decreasing pixel dimensions was also fabricated (Fig. 1c,ii) highlighting that this method is able to perform consistently across a range of pixel dimensions.

With the approach shown here, a single lithographic step is used and a single material combination is required (Agresist- $\mathrm{Ag}$ ) resulting in relatively narrowband and high transmission spectra. Given the potential for translation into conventional photolithography with grayscale Cr masks, our approach has the potential to reach the realms of larger volume manufacturing.

This work was supported by Cancer Research UK (CRUK, C47594/A24669, C14303/A17197, C47594/A16267)

\section{References}

[1] B.E. Bayer, Color imaging array, U.S. Patent No. 3971065. 1976

[2] P.J. Lapray et al., Multispectral filter arrays: recent advances and practical implementation, Sensors, 14(11), 21626-21659, 2014

[3] A. Kristensen et al., Plasmonic color generation, Nat. Rev. Mat., 2, 16088, 2016

[4] P. Genevet et al., Recent advances in planar optics: from plasmonic to dielectric metasurfaces, Optica, 4(1), 139, 2017

[5] Q. Chen el al., Nanophotonic image sensors. Small, 12, 4922-4935, 2016

[6] C. Williams et al., Single-step fabrication of thin-film linear variable bandpass filters based on metal-insulator-metal geometry, Appl. Opt.

$55,9237-924,2016$ 